\title{
The effects of tilted outline frames and intersecting line patterns on judgments of vertical*
}

\author{
PETER M. WENDEROTH \\ University of Sydney, Sydney, Australia, 2006
}

\begin{abstract}
The angular function of the rod-and-frame illusion has been attributed to the effects of the major, virtual axes of the frame. Only one of two possible interpretations of this hypothesis adequately accounted for vertical settings made in the presence of outline frames and intersecting line patterns tilted between vertical and 45 deg. The results obtained with intersecting line patterns did not appear to be explicable by torsional effects or the simple addition of tilted-line illusions.
\end{abstract}

When a rod is enclosed in a tilted square outline frame, the rod usually has to be slightly tilted in order to be judged vertical (Witkin \& Asch, 1948). The magnitude and direction of this rod-and-frame illusion (RFI) depends on the degree of frame tilt. Beh, Wenderoth, and Purcell (1971) found that clockwise frame tilts of 15 and 60 deg produced clockwise errors in vertical settings but that anticlockwise errors occurred with clockwise frame tilts of 30 and $75 \mathrm{deg}$. At other frame positions $(0,45$, and $90 \mathrm{deg})$, settings were close to gravitational vertical. These results were consistent with the hypothesis that the rod was set towards whichever of the four major frame axes (vertical, horizontal, and two diagonals) was nearest to gravitational vertical. Further support for this hypothesis was obtained when frame shape was varied (triangle, hexagon, rectangle), and the positions of the major axes correctly predicted the direction of rod settings for each frame shape at each tilt (Beh \& Wenderoth, 1972).

Previous experiments have thus been concerned with the effects of outline figures, where the major-axes hypothesis (MAH) makes clear predictions about the effects of virtual axes. The present experiment set out to test the effect of physically present axes in the absence of an outline shape (Fig. 1). For this rod-and-axes illusion (RAI), MAH can predict two different results, depending on its interpretation.

The first interpretation (MAH-1) is that the four virtual axes of an outline square have an effect equivalent to four actual contours, such as those in Fig. 1. In that event, the direction, though not necessarily the magnitude, of RAI and RFI should be the same for a given inducing-figure (IF) tilt. Since the axes are $45 \mathrm{deg}$ apart, the direction of illusion should

*This research was supported by University of Sydney research grants, 1971-1972. The author wishes to thank the University for its support and also the following for their assistance: Ian Curthoys, for many things, Ralph Hall, R. S. Roger, and G. Coleman, for statistical advice, and, in particular, Stewart Dunn, who most ably and intelligently assisted in testing some Ss. The apparatus was remodelled from a standard rod-and-frame stimulus originally constructed by $\mathrm{G}$. Whiteman, who also built the shutter, biteboard, and UV light box. alternate every $22.5 \mathrm{deg}$ of IF tilt, with no illusion when one of the axes is at vertical (IF tilts of 0 and $45 \mathrm{deg}$ ) or when two axes are equidistant from vertical (22.5-deg IF tilt).

The second interpretation (MAH-2) is that any IF, whether an outline or axes figure, has virtual axes which determine its inducing effect. In that case, a four-axis figure (e.g., Fig. 1) has eight major axes or axes of symmetry (see Beh \& Wenderoth, 1972). Four of these are the axis contours themselves, while four others are virtual axes which lie along the angle bisectors (Fig. 2A). Since these eight axes are spaced by $22.5 \mathrm{deg}$, MAH-2
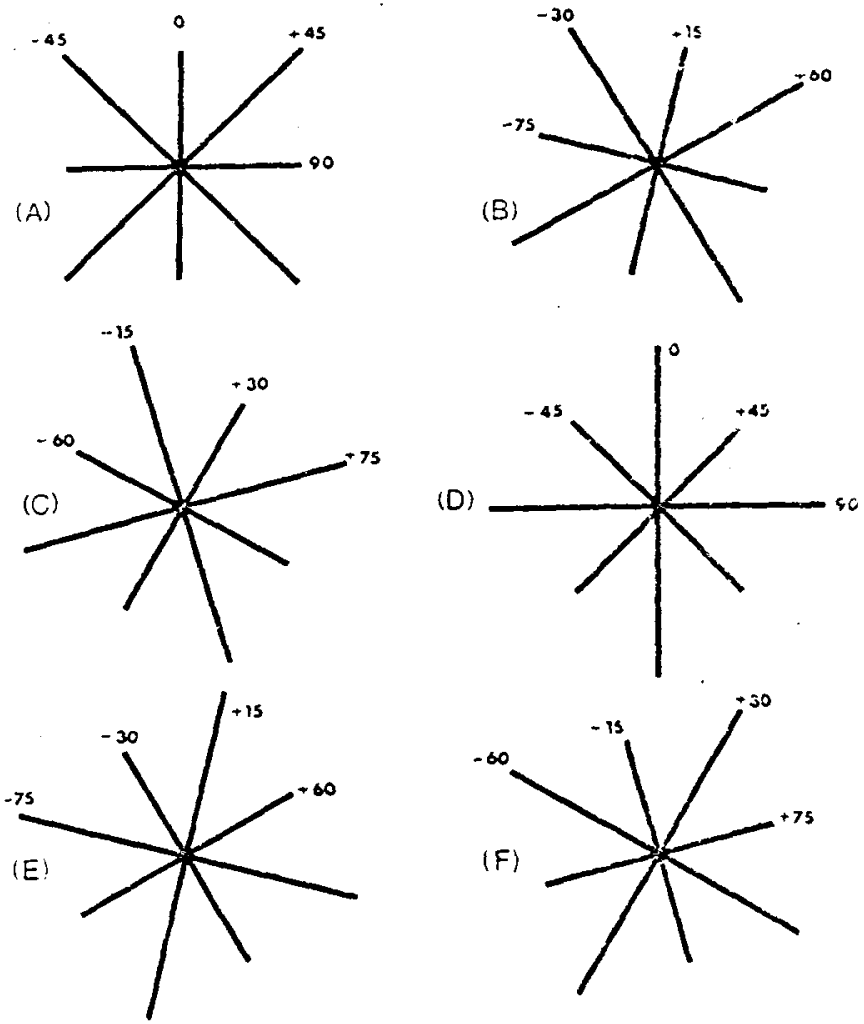

Fig. 1. Orientations of four component lines of IF for RAI illusion, as IF tilt varies through $0 \mathrm{deg}$ (A), $15 \mathrm{deg}$ (B), $30 \mathrm{deg}$ (C), $45 \mathrm{deg}$ (D), $60 \mathrm{deg}(\mathrm{E})$, to $75 \mathrm{deg}(\mathrm{F})$. 


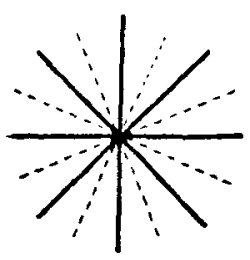

A.

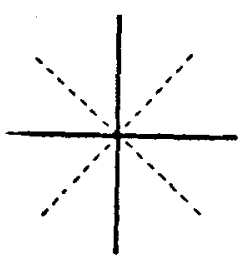

C.

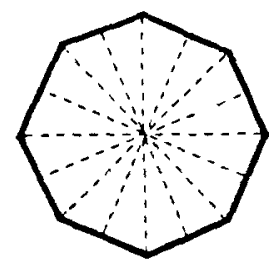

B.

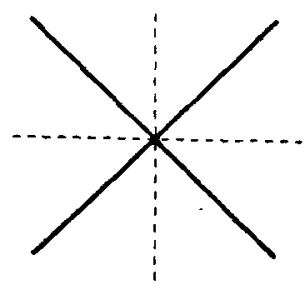

D.
Fig. 2. (A) An IF for RAI illusion, showing hypothetical inhibitory zones (dotted) along angle bisectors. (B) Octagon outline frame showing eight major frame axes. (C) Vertical-horizontal axis figure, and (D) diagonal axis figure, both with virtual axes dotted along angle bisectors.

predicts that RAI should alternate in direction every $11.25 \mathrm{deg}$, twice the frequency predicted by MAH-1. The outline figure that has eight virtual major axes spaced $22.5 \mathrm{deg}$ is an octagon (Fig. 2B). Hence, MAH-2 predicts the same direction of vertical settings for an octagonal frame and RAI at any IF tilt. On the other hand, MAH-1 predicts the same direction of vertical settings for RAI and a square frame at any IF tilt. In this experiment, vertical settings were obtained for clockwise IF tilts from 0 to $45 \mathrm{deg}$. Within that range, MAH predicts that the angular function of a square frame should describe a cubic function, with clockwise settings from 0 to $22.5 \mathrm{deg}$, anticlockwise settings from 22.5 to $45 \mathrm{deg}$, and no illusion at $0,22.5$, and $45 \mathrm{deg}$. Since MAH-1 predicts the same angular function for RAI and a square frame, it predicts a cubic function for RAI.

An outline octagon should describe a quintic function, with clockwise settings from 0 to $11.25 \mathrm{deg}$ and 22.5 to $33.75 \mathrm{deg}$, anticlockwise settings from 11.25 to $22.5 \mathrm{deg}$ and 33.75 to $45 \mathrm{deg}$, with no effect occurring at $0,11.25,22.5,33.75$, and $45 \mathrm{deg}$. Since MAH-2 predicts the same angular function for RAI and an octagonal frame, it predicts a quintic function for RAI.

As a further test of the two hypotheses, two two-axis figures were included in the experiment (Fig. 2C, D). According to MAH-1, only the physically present contours affect settings, so that, in both cases, errors in vertical settings will be unidirectional between 0 - and 45-deg IF tilts. In one case (Fig. 2C), the nearest axis will always be clockwise as the IF tilts from 0 to $45 \mathrm{deg}$, so that deviations from vertical should always be clockwise. In the other case (Fig. 2D), settings should be anticlockwise. Hence, MAH-1 predicts that vertical settings will follow a quadratic function for both these IFs. Since MAH-2 predicts that the dotted virtual axes will be effectual, it predicts a cubic function for these two IFs. The six IFs used in the experiment are shown in Fig. 3 (Groups 1 and 2 were both tested with the frame, the second group merely providing a replication). The two forms of four-axis figure (Groups 5 and 6) were included because the effect of the virtual outline was not known: Although MAH-1 predicts a cubic trend for both groups and MAH-2 predicts a quintic trend for both groups, it is possible that different effects occur, depending on whether the end points of the RAI figure describe an octagon (Group 5) or a square (Group 6).

A summary of the trends predicted by MAH-1 and MAH-2 for the angular functions of each of the IFs from 0- to 45-deg IF tilt is given in Table 1.

\section{METHOD}

\section{Subjects}

Seventy students from an introductory psychology course acted as Ss, 25 males and 45 females. The alignment test indicated that $48 \mathrm{Ss}$ were right-eye dominant and 22 were left-eye

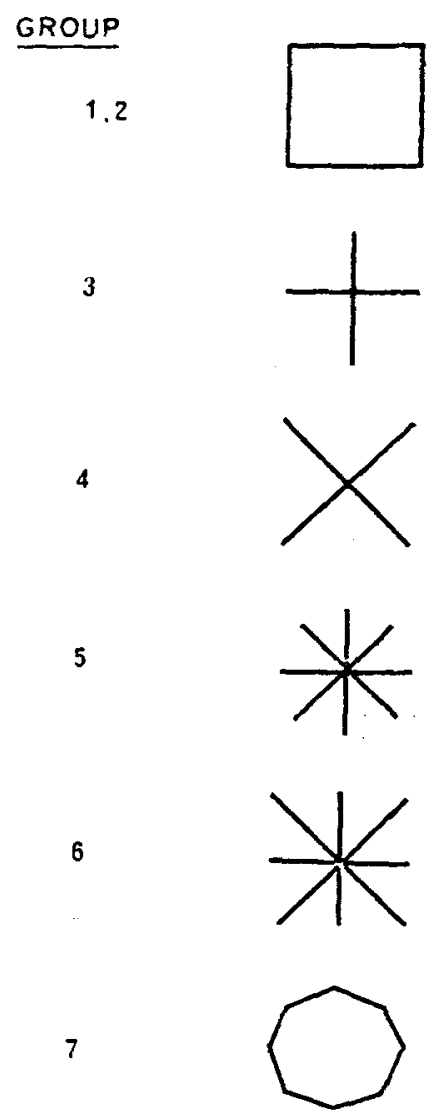

Fig. 3. IFs for Goups 1-7. Group 2 is a replication of Group 1. 
Table 1

Predicted Trend Components of Angular Functions of Vertical Settings Induced by Different IFs (IF Tilts: 0-45 Deg)

\begin{tabular}{lcll}
\multicolumn{1}{c}{ IF } & Group & MAH-1 & MAH-2 \\
\hline Square Frame & 1,2 & Cubic & Cubic \\
Two-Axis Figures & 3,4 & Quadratic & Cubic \\
Four-Axis Figures & 5,6 & Cubic & Quintic \\
Octagon & 7 & Quintic & Quintic \\
\hline
\end{tabular}

dominant. Any S who normally wore corrective lenses did so in the experiment.

\section{Experimental Design}

The $70 \mathrm{Ss}$ were assigned randomly to seven groups of 10 each. Each group of Ss made vertical settings for only one of the IFs (Fig. 3). Within a group, each of the Ss made four judgments of vertical under each of 19 conditions of clockwise (+) IF tilt: 0 (vertical) to $45 \mathrm{deg}$, in 2.5 -deg steps. Settings at a particular IF tilt were from four starting positions (SPs): \pm 10 and \pm 15 deg. Each $S$ made the 76 judgments in a different random order, determined by shuffling a pack of cards.

\section{Apparatus}

A 28-cm-long rod and a 58-cm-square wooden backplate were mounted on a table. The plate, which served as a holder for the various IFs, was coaxially pivoted with the rod by means of concentric shafts and bushes so that the two could be independently tilted (Fig. 4). The IFs were prepared on sheets of heavy $60-\mathrm{cm}$-square pasteboard which were drilled in the center and at the corners. Each such sheet could be mounted on the backplate so that pins at the corners of the plate pierced the drilled corners of the pasteboard, enabling any IF to be removed and replaced without shifting its position on the frame. When an IF sheet was fixed to the backplate, the shaft for the rod terminated flush with the drilled center of the pasteboard. This shaft was tapped so that the rod could be connected or removed easily by means of a brass bolt soldered perpendicularly to the center of the rod.

Special care was taken that the rod not slip and that all IF lines have the required orientation when mounted. Small nylon washers on the bolt of the rod fixed it firmly in position. All IF lines were constructed with reference to a plumb line fixed to the ceiling and suspended in oil below the apparatus. The plumb line, which was removed during experimental trials, was used to check rod position systematically throughout each test session to ensure that it maintained its setting.

Either $E$ or $S$ could control rod movement by switches connected to a $2 \mathrm{deg} / \mathrm{sec}$ synchronous motor. Tilt of the IF backplate was varied manually by $E$. The angles of IF and TF tilt were read from a small and large protractor, respectively, to the nearest 0.5 and $0.25 \mathrm{deg}$.

A dental composition biteboard, foreheadrest, and monocular viewing tube gave $S$ a fixed right-eye view of the stimuli. A solenoid-operated shutter at the distal end of the viewing tube opened only when $E$ switched rod control to $S$ and closed when rod control was switched to $E$.

Stimuli were illuminated by an ultraviolet light placed on a table directly in front of S's occluding screen.

\section{Stimuli}

The IFs were prepared by laying $2.5-\mathrm{mm}$-wide $(3.5 \mathrm{~min}$ ) strips of pink adhesive vinyl tape (Sellotape) on the pasteboard sheets, which had been painted flat black. The horizontal width of all IFs was $30.5 \mathrm{~cm}$ ( $7 \mathrm{deg} 6 \mathrm{~min})$.

The 28-cm-long (6 deg $33 \mathrm{~min}$ ) cylindrical steel rod was painted with Gestetner correction fluid to a thickness of 2-2.5 mm. It was found that not only were the vinyl tape and the fluid sensitive to ultraviolet light, but that both fluoresced a similar, even shade of deep pink. The luminance of all display contours was about $.03 \mathrm{~mL}$ (SEI photometer).

\section{Procedure}

A $1 \frac{1}{2}-\mathrm{h}$ session was required to complete preliminaries (eye-dominance test, making biteboard aligning $S$, and
Fig. 4. The apparatus. Rod and frame not drawn to scale.

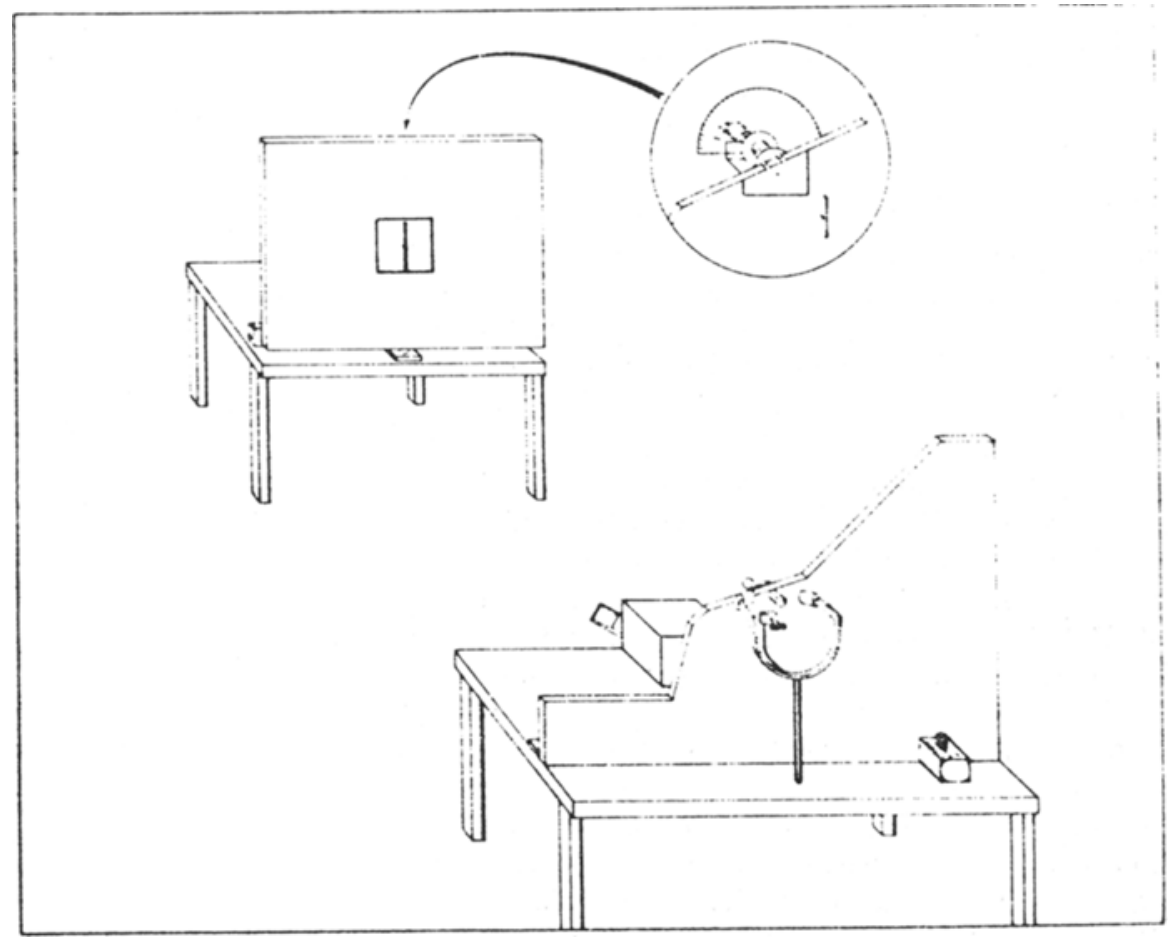



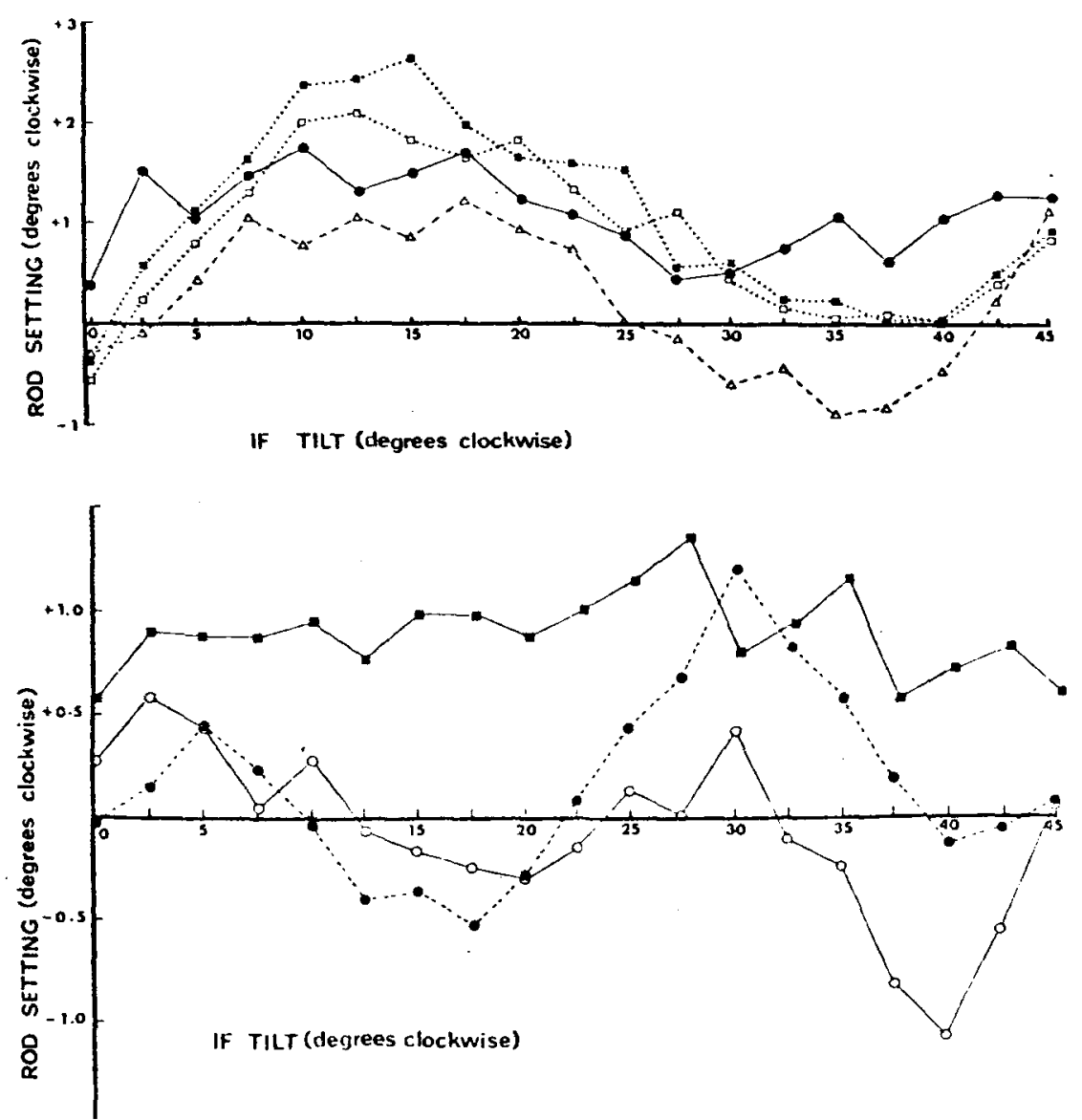

Fig. 5. Vertical settings as a function of IF tilt, Groups 1-4. Group 1, open squares; Group 2, solid squares; Group 3, solid circles; and Group 4, open triangles.
Fig. 6. Vertical settings as a function of IF tilt, Groups 5-7. Group 5, open circles; Group 6, solid squares; and Group 7, solid circles. instruction) and to run the 76 trials in four blocks of 19 trials with a 2 -min rest between blocks. The adjustment method with bracketing was used, and $S$ was required to fixate a small black spot (unpainted) at the center of the rod at all times. The instruction was to set the rod to gravitational vertical, explained by means of the plumb line, within a time of $10-15$ sec. Since the large number of trials plus the required fixation were quite exhausting, $S$ was instructed to request unscheduled rest periods if necessary.

Four practice trials were given with the IF vertical, and $S$ was told once again that the required setting was gravitational vertical, a setting which was to be evaluated independently of the positions of any of the background contours.

\section{RESULTS}

Mean rod settings judged gravitationally vertical are plotted as a function of IF tilt for Groups 1-4 (Fig. 5) and Groups 5.7 (Fig. 6).

Tests of trend were carried out on each group, using a version of Grant's (1956) analysis and a method of unplanned tests (Rodger, 1967a, b). ${ }^{1}$ A summary of the major tests of trend is shown in Table 2.

Comparing these results with the predictions of MAH-1 and MAH-2 (Table 1), it is clear that the results favor MAH-2. Both MAH-1 and MAH-2 predicted cubic trend in Groups 1 and 2 and quintic trend in Group 7. These common predictions were confirmed. However, MAH-1 predicted quadratic trend in Groups 3 and 4 and cubic trend in Groups 5 and 6 . None of those predictions was confirmed. On the other hand, MAH-2 correctly predicted cubic trend in Groups 3 and 4 and quintic trend in Group 5, though the predicted quintic trend in Group 6 did not occur. Neither version of the MAH hypothesis predicted the obtained linear trend in Group 2 or the quadratic trend in Groups 1 and 2.

The apparent mean difference between Group 6 and Groups 5 and 7 (Fig. 6) was not detected [F.95 7(63) $=$ $1.23: F=0.57]$. The failure to detect the difference is probably due to the lack of power of tests between Ss in split-plot designs (the error term for between-group

Table 2

Summary of Tests of Trend, Linear to Quintic, for Groups 1.7*

\begin{tabular}{cccccc} 
& \multicolumn{5}{c}{ Trend Component } \\
Group & Linear & $\begin{array}{c}\text { Quad- } \\
\text { ratic }\end{array}$ & Cubic & Quartic & Quintic \\
\hline 1 & $\mathrm{A}(0.74)$ & $\mathrm{R}(1.92)$ & $\mathrm{R}(5.15)$ & $\mathrm{A}(0.08)$ & $\mathrm{A}(0.01)$ \\
2 & $\mathrm{R}(1.74)$ & $\mathrm{R}(2.01)$ & $\mathrm{R}(6.86)$ & $\mathrm{A}(0.01)$ & $\mathrm{A}(0.06)$ \\
3 & $\mathrm{~A}(0.16)$ & $\mathrm{A}(0.00)$ & $\mathrm{R}(1.48)$ & $\mathrm{A}(0.27)$ & $\mathrm{A}(0.08)$ \\
4 & $\mathrm{~A}(0.89)$ & $\mathrm{A}(0.08)$ & $\mathrm{R}(5.81)$ & $\mathrm{R}(1.28)$ & $\mathrm{A}(0.01)$ \\
5 & $\mathrm{~A}(1.08)$ & $\mathrm{A}(0.01)$ & $\mathrm{A}(0.01)$ & $\mathrm{A}(0.35)$ & $\mathrm{R}(2.13)$ \\
6 & $\mathrm{~A}(0.00)$ & $\mathrm{A}(0.15)$ & $\mathrm{A}(0.00)$ & $\mathrm{A}(0.00)$ & $\mathrm{A}(0.12)$ \\
7 & $\mathrm{~A}(0.18)$ & $\mathrm{A}(0.03)$ & $\mathrm{A}(1.05)$ & $\mathrm{A}(0.11)$ & $\mathrm{R}(3.00)$ \\
\hline
\end{tabular}

*In each cose. critical ralue of $F .95^{7}(63)=1.23 . A=$ accept $H_{O}: R=$ reject $H_{O}$. Obtained $F$ ralle in parentieses. 
Fig. 7. Vertical settings as a function of IF tilt, Group 3 (bottom) and Group 4 (top). SPs are: +15 deg (solid circles), $+10 \mathrm{deg}$ (solid triangles), -10 deg (open circles), and $-15 \mathrm{deg}$ (open triangles).

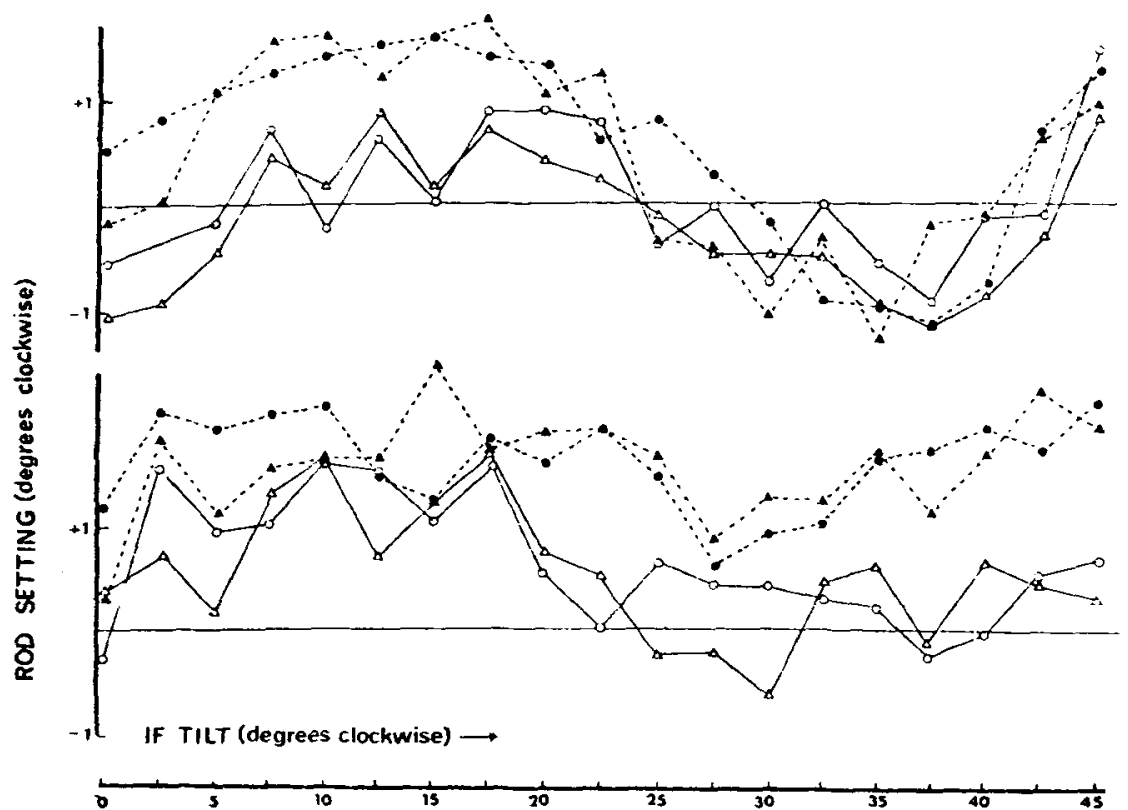

mean comparisons was 46.07 , while that for betweengroup trends was 2.69 ).

Despite the overall similarity in the results of Groups 1.4, there are differences, in particular the quadratic trend in Groups 1 and 2 and, from a further test, a difference between Groups 3 and 4 in quartic trend $(F=1.36)$. A part of these differences appears to be due to an interaction between the nature of the IF and starting position (SP) effects. Generally, SP regression effects are marked when an isolated TF is set to the vertical (Wenderoth et al, 1968). The IFs in Groups 3 and 4 add the complication that they both have line components which, at certain angles of IF tilt, are close to or exactly at gravitational vertical. In such cases, particularly when $O$ is permitted to bracket, it is possible that SP effects could be overridden by O's tendency to align the TF with the vertical IF component or to judge vertical relative to the near-vertical IF component. If so, SP regression effects would be mainly restricted to IF tilts in which all line components were well removed from the true vertical. The essential point here is that SP regression effects should therefore occur for Group 4 mainly at IF tilts near vertical but for Group 3 at IF tilts near $45 \mathrm{deg}$. Figures $7 \mathrm{~A}$ and $7 \mathrm{~B}$ show that this was the case. The interaction between SP regression and IF tilt for the frame IFs was similar to that for Group 3, not Group 4. This is to be expected if the vertical or near-vertical sides of the frame are used for alignment at IF tilts close to vertical.

In brief, an interaction between SP regression effects and IF tilts may account, in part, for the differences obtained between Groups 3 and 4 and the differences between these two groups and Groups 1 and 2 .

\section{DISCUSSION}

The Beh et al (1971) major-axes hypothesis predicted the results obtained with the octagonal outline frame in this experiment. In an attempt to predict the results for four-axis (RAI) and two-axis IFs, two forms of the hypothesis (MAH-1 and MAH-2) were distinguished. Only MAH-2 successfully predicted the general results of the experiment, which suggests that virtual axes affect vertical settings made in the presence not only of outline frames, but also of intersecting line IF patterns. There are, however, aspects of the results for which the hypothesis appears not to account.

The RFI in both Groups 1 and 2 was +ve (clockwise at every IF tilt, with means for the two groups of +0.88 and +1.09 , respectively. The hypothesis predicts a function which has a mean of zero, symmetrical around an IF tilt of $22.5 \mathrm{deg}$. The predicted symmetry did occur: averaging over the two groups, the mean settings were $+2.26 \mathrm{deg}$ at $15-\mathrm{deg}$ IF tilt, $+1.47 \mathrm{deg}$ at $22.5-\mathrm{deg}$ IF tilt, and $+0.54 \mathrm{deg}$ at $30-\mathrm{deg}$ IF tilt. The average of the effects at $15-$ and $30-\mathrm{deg}$ IF tilts was $+1.40 \mathrm{deg}$, close to the +1.47-deg setting at 22.5-deg IF tilt.

The fact that pretests were not used in this experiment is a possible explanation of the positive shift, though Beh et al (1971) did not use pretests and obtained a significant negative effect at 30 -deg frame tilt. However, they allowed Os to use the preferred eye, and they also rejected Os whose settings were not within \pm 2 deg of zero at 0 -deg IF tilt. In the present study, only the right eye was used, and it appears that vertical settings of an isolated rod are significantly more clockwise with the right than with the left eye (Schneider, 1966). Thus, at frame tilts other than zero, where reference lines are not available, not only SP effects, but also eye effects might be expected to occur. The individual SP functions for the frame, which closely resemble those of Group 3 (Fig. 7) are consistent with this view.

With reference to the RAI; MAH-2 predicted quintic 
trend for both Group 5 and Group 6, but no significant trend occurred in Group 6. The two most obvious differences between the IFs is that their outlines differ, and one has axes of different length (Group 5-outline octagon, axes all equal; Group 6-outline square, diagonal axes longer). Thus, MAH may require some incorporation of the effects of line length or virtual outline. It may be relevant to note that the Group 6 result resembles the average of the frame and octagon results and the Group 6 IF has the major axes of one and the outline of the other. If axis length is an important variable, some of the difference between Groups 3 and 4 could be due to that factor. ${ }^{2}$

Two effects which are frequently discussed in relation to errors in vertical settings induced by tilted IF lines are eye torsion (Howard \& Templeton, 1966) and tilt aftereffect and illusion (Gibson, 1937; Gibson \& Radner, 1937).

It is extremely unlikely that torsional effects can explain the present results, for the following reasons. First, the two two-axis figures gave similar results; an explanation purely in terms of torsion induced by tilted IF lines would predict almost opposite results, for when both figures are vertical one has its component lines at 0 and $90 \mathrm{deg}$, the other at $\pm 45 \mathrm{deg}$. Second, significant torsional effects have usually been found only with moving stimuli (Howard \& Templeton, 1964; Hughes, Brecher, \& Fishkin, 1972); torsion induced by the moving TF would therefore have to be assumed to interact in different and complex ways with the different IF lines in this study. In particular, since torsion appears to increase monotonically with amplitude of line rotation (Howard \& Templeton, 1964), the quintic variation of rod settings induced by the four-axis figure would be difficult to explain, as would the cubic trend in the two-axis data. Hughes et al (1972) report on significant pretest-posttest differences in vertical settings induced by a stationary 16-spoke IF, but they do not, unfortunately, report its orientation.

If an explanation of the results were sought in terms of Gibson-type tilt effects, the simplest initial hypothesis would be that each line of an axis figure independently induces an illusion and that all illusions so induced simply add. The present results falsify this hypothesis. Consider, for example, the two-axis IF of Group 4. When this IF is tilted 15 deg clockwise, one of its arms is tilted clockwise $60 \mathrm{deg}$ and the other is tilted anticlockwise $30 \mathrm{deg}$. A single line or a grating tilted $60 \mathrm{deg}$ clockwise induces an illusion or aftereffect which results in anticlockwise errors in vertical settings, and a single line or grating tilted $30 \mathrm{deg}$ anticlockwise should also induce anticlockwise settings, for, at IF tilts less than about $50 \mathrm{deg}$, induced errors in vertical settings are in the same direction as IF tilt (Gibson \& Radner, 1937; Logan, 1962; Morant \& Harris, 1965: Wallace \& Crampin, 1969; Muir \& Over, 1970: White, 1971). Since the effect obtained with Group 4 at 15-deg clockwise IF tilt was a clockwise ( + ) setting of $0.89 \mathrm{deg}$. the summation of two anticlockwise (-) effects cannot explain the result. The use of the right eye only may have counteracted the - ve effect, but not sufficiently to produce the obtained effects. In fact, the relevant experiment has been conducted with 14 Os, each tested under three IF conditions. A single-line IF at $+60 \mathrm{deg}$ gave a -0.39 -deg mean setting. A similar IF at $-30 \mathrm{deg}$ gave a -0.08 -deg setting. The combination of these two IFs gave a mean setting of $+0.44 \mathrm{deg}$. The null hypothesis that the third mean was the sum of the other two was rejected $\left[F_{.95}(1,26)=4.23 ; F=4.99\right]$. Finally, the additive illusion hypothesis, when applied to RAI (Fig. 1), produces predictions identical to those of MAH-1, which has been rejected.

It is possible that MAH-2 may be expressed alternately in terms of inhibitory interaction between orientation detectors in the visual cortex (e.g., Blakemore et al, 1970; Bouma \& Andriessen, 1968, 1970; Lennie, 1971; Nelson, 1969), but such speculation may be premature (Uttal, 1971).

\section{REFERENCES}

Beh, H. C., \& Wenderoth, P. M. The effect of frame shape on the angular function of the rod-and-frame illusion. Perception \& Psychophysics, 1972, 11, 35-37.

Beh, H. C., Wenderoth, P. M., \& Purcell, A. T. The angular function of a rod-and-frame illusion. Perception \& Psychophysics, 1971, 9, 353-355.

Blakemore, C., Carpenter, R. H. S., \& Georgeson, M. A. Lateral inhibition between orientation detectors in the human visual system. Nature, 1970, 228, 37-39.

Bouma, H., \& Andriessen, J. J. Induced changes in the perceived orientation of line segments. Vision Research, 1970, 10, 333-349.

Gibson, J. J. Adaptation, after-effect and contrast in the perception of tilted lines: II. Simultaneous contrast and the areal restriction of the after-effect. Journal of Experimental Psychology, 1937, 20, 553-569.

Gibson, J. J., \& Radner, M. Adaptation, aftereffect and contrast in the perception of tilted lines: I. Quantitative studies. Journal of Experimental Psychology, 1937, 20, 453-467.

Grant, D. A. Analysis of variance tests in the analysis and comparison of curves. Psychological Bulletin, 1956, 53, 141-154.

Howard, I. P., \& Templeton, W. B. Visually-induced eye torsion and tilt adaptation. Vision Research, 1964, 4, 433-437.

Howard, I. P., \& Templeton. W. B. Human spatial orientation. London: Wiley, 1966.

Hughes, P. C., Brecher, G. A., \& Fishkin, S. M. Effects of rotating backgrounds upon the perception of verticality. Perception \& Psychophysics, 1972, 11, 135-138.

Lennie, P. Distortions of perceived orientation. Nature. 1971. 233, 155-156.

Logan, J. A. An examination of the relationship between visual illusions and figural after-effects. Unpublished doctoral dissertation, University of Sydney, 1962.

Morant, R. B., \& Harris. J. R. Two different aftereffects of exposure to visual tilts. American Journal of Psychology. 1965, 78, 218-226.

Muir, D., \& Over, R. Tilt aftereffects in central and peripheral vision. Journal of Experimental Psychology. 1970. 85. 165-170.

Nelson, J. The misperception of contour. Unpublished manuscript. State University of New York. Stony Brook. 1969. 
Rodger, R. S. Type I errors and their decision basis. British Journal of Mathematical \& Statistical Psychology, 1967a, 20, $51-62$.

Rodger, R. S. Type II errors and their decision basis. British Journal of Mathematical \& Statistical Psychology, 1967b, 20, 187-204.

Schneider, C. W. Monocular and binocular perception of verticality and the relationship of ocular dominance. American Journal of Psychology, 1966, 79, 632-636.

Uttal, W. R. The psychobiological silly season - or - what heppens when neurophysiological data become psychological theories. Journal of General Psychology, 1971, 84, 151-166.

Wallace, G. K., \& Crampin, D. J. The effect of background density on the Zöllner illusion. Vision Reserach, 1969, 9, 167-177.

Wenderoth, P. M., Rodger, R. S., \& Curthoys, I. S. Confounding of psychophysical errors and sensory effects in adjustment measures of spatial aftereffects. Perception \& Psychophysics, $1968,4.133-138$.

White, K. G. Zöllner illusion as perceptual enlargement of acute angles. Psychonomic Science, 1971, 24, 73-75.

Witkin, H. A., \& Asch, S. E. Studies in space orientation. IV. Further experiments on perception of the upright with displaced visual fields. Journal of Experimental Psychology, $1948 b, 38,762-782$.

\section{NOTES}

1. The $F$ values for this analysis, $F(1-\alpha)^{\nu_{1}}$ are given in Rodger, R. S. Intermediate statistics. Sydney: University Cooperative Bookshop, 1965. In this paper, F.957(63) means the critical value of $F$ for $\alpha=0.05$ with $\nu_{1}=7$ and $\nu_{2}=63$.

2. If a major axis is strictly defined as one of bilateral symmetry (producing mirror images), then the Group 6 IF has only the four major axes of an outline frame, i.e., the axes themselves. The virtual angle bisectors cut the figure into reverse mirror images, which produce point or rotational symmetry only.

(Received for publication October 25, 1972; revision received February 12, 1973.) 